Note

\title{
Effects of Various Amino Acids on Methionine-Induced Hyperhomocysteinemia in Rats
}

\author{
Shin-ichiro Fukada, Tatsuya Morita, and Kimio SugiYama ${ }^{\dagger}$ \\ Department of Applied Biological Chemistry, Faculty of Agriculture, Shizuoka University, \\ 836 Ohya, Suruga-ku, Shizuoka 422-8529, Japan
}

Received December 28, 2007; Accepted April 29, 2008; Online Publication, July 7, 2008

[doi:10.1271/bbb.70833]

Rats were fed diets supplemented with $1 \%$ L-methionine with and without $2.5 \%$ various amino acids for $7 \mathrm{~d}$ to determine what amino acids other than glycine, serine, and cystine can suppress methionine-induced hyperhomocysteinemia. L-Glutamic acid, L-histidine, and L-arginine significantly suppressed methionineinduced enhancement of plasma homocysteine concentrations, but the mechanisms underlying the effect of these amino acids are thought not to be identical.

Key words: homocysteine; methionine; glutamic acid; histidine; arginine

It is widely recognized that increased plasma homocysteine concentration is an independent risk factor for cardiovascular disease. ${ }^{1,2)}$ The plasma homocysteine concentration is affected by various factors, including genetic, nutritional, physiological, clinical, lifestyle, and drugs. ${ }^{1,2)}$ For instance, genetic defects in several enzymes that participate in the metabolism of homocysteine cause hyperhomocysteinemia due to impaired homocysteine metabolism. Deficiencies of some vitamins, such as folate and vitamin $\mathrm{B}_{6}$ and $\mathrm{B}_{12}$ also bring about increases in the plasma homocysteine concentration. Furthermore, higher plasma homocysteine concentrations are associated with older age, male sex, impaired renal function, and high alcohol intake. It was confirmed that some amino acids participate in the metabolism of methionine or homocysteine (Fig. 1). Verhoef et al. $^{3)}$ have found that serine and cystine suppress the enhancement of plasma homocysteine concentration due to a single ingestion of a methionine-supplemented low-protein meal in humans. In addition, we found that dietary supplementation with glycine and with serine suppresses methionine-induced hyperhomocysteinemia in rats, ${ }^{4}$ but little information is available on the effects of amino acids other than glycine, serine, and cystine on plasma homocysteine concentration.

In this study, we investigated the effects of 19 amino acids, which were individually added to the diet, on

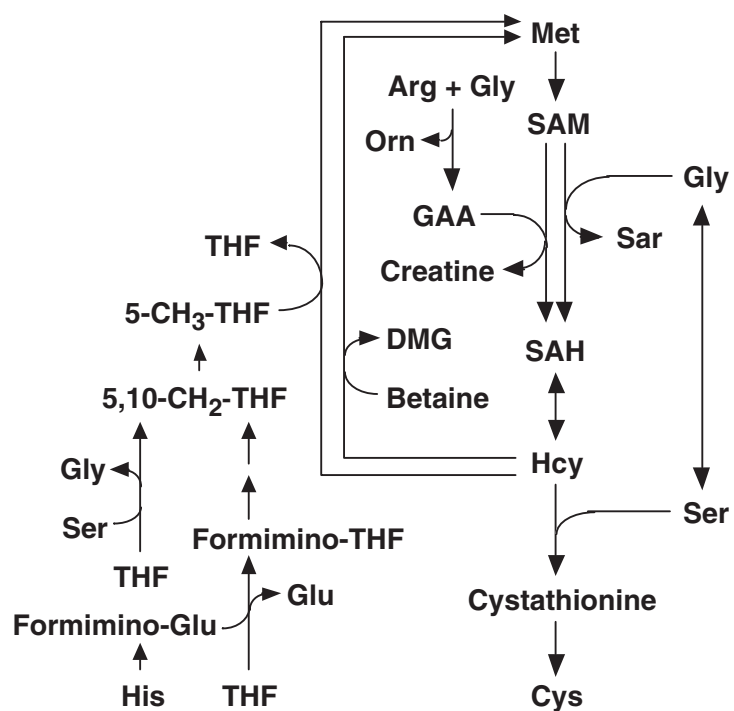

Fig. 1. Interrelation between Methionine Metabolism and Some Other Amino Acids.

DMG, $N, N$-dimethylglycine; GAA, Guanidinoacetic acid; Hcy, homocysteine; SAH, $S$-adenosylhomocysteine; SAM, $S$-adenosylmethionine; Sar, sarcosine; THF, tetrahydrofolic acid.

hyperhomocysteinemia induced by dietary supplementation with methionine in rats to determine by what amino acids the increase in the plasma homocysteine concentration can be suppressed.

Male 6-week-old rats (120-140 g) of the Wistar strain were obtained from Japan SLC (Hamamatsu, Japan). The rats were fed a $25 \%$ casein $(25 \mathrm{C})$ diet for $5 \mathrm{~d}$ and then they were fed the $25 \mathrm{C}$ or an experimental diet for $7 \mathrm{~d}$. The composition of the $25 \mathrm{C}$ diet was as follows (g/100 g): casein, 25; corn starch, 43.25; sucrose, 20; corn oil, 5; AIN-93G mineral mixture, 3.5; AIN-93G vitamin mixture, 1 ; choline bitartrate, 0.25 ; cellulose, 2 . The control diet $(25 \mathrm{CM})$ was $25 \mathrm{C}+1 \%$ L-methionine, and the experimental diets were $25 \mathrm{CM}+2.5 \%$ amino acid (one of the 19 amino acids). The amino acids, all L-isomers except for glycine, were added to the diet at the expense of starch. After the rats were given free access to water and the experimental diets for $7 \mathrm{~d}$, they

\footnotetext{
$\dagger$ To whom correspondence should be addressed. Fax: +81-54-238-4877; E-mail: acksugi@agr.shizuoka.ac.jp
} 
were killed by decapitation between 10:00 and 11:00 h to obtain the blood and liver. The experimental plan was approved by the Laboratory Animal Care Committee of the Faculty of Agriculture at Shizuoka University.

After the collection of blood, the whole liver was quickly removed, cut into two portions, weighed, and quickly frozen in liquid nitrogen and stored at $-80^{\circ} \mathrm{C}$ until needed for analysis. The homogenization of the liver and following centrifugation was essentially by the method described previously. ${ }^{4)}$ The concentrations of total homocysteine and cysteine in the plasma were measured by HPLC following Durand et al. ${ }^{5)}$ The concentrations of $S$-adenosylmethionine (SAM) and $S$-adenosylhomocysteine (SAH) in the liver were measured by HPLC, essentially following Cook et al. ${ }^{6}$ The activity of cystathionine $\beta$-synthase (E.C. 4.2.1.22) (CBS) in the liver was measured following Mudd et al. ${ }^{7)}$ but HPLC was used in the assay of the reaction product cystathionine, following Einarsson et al. ${ }^{8)}$ Data were expressed as the mean \pm SEM. Student's $t$-test was used to analyze statistical differences between the control and experimental groups.

Body weight gain $(\mathrm{g} / 7 \mathrm{~d})$ was significantly lower in the rats fed diets supplemented with alanine $(26 \pm 1)$, leucine $(23 \pm 1)$, isoleucine $(26 \pm 1)$, threonine $(26 \pm 1)$, cysteine $(12 \pm 2)$, or histidine $(19 \pm 1)$ than in the rats fed the $25 \mathrm{CM}$ diet $(31 \pm 2)$ at $p<0.05$, whereas food intake $(\mathrm{g} / 7 \mathrm{~d})$ was significantly lower in the rats fed the diet supplemented with valine $(73 \pm 1)$ than in the rats fed the $25 \mathrm{CM}$ diet $(85 \pm 4)$ at $p<0.05$. The plasma homocysteine concentration was markedly higher in the rats fed the $25 \mathrm{CM}$ diet than in those fed the 25C diet (Fig. 2A). The methionine-induced increase in the plasma homocysteine concentration was significantly suppressed by glutamic acid, histidine, and arginine in addition to glycine and serine, although the effects of glutamic acid, histidine, and arginine were apparently smaller than the effects of glycine or serine. In contrast, the plasma cysteine concentration, which was measured for comparison, was less affected by methionine and the other amino acids, although some amino acids significantly decreased and some others significantly increased the plasma cysteine concentration (Fig. 2B). Hepatic concentrations of SAM, SAH and homocysteine were significantly higher in the rats fed the $25 \mathrm{CM}$ diet than in those fed the $25 \mathrm{C}$ diet, and the increases in these methionine metabolite concentrations were significantly suppressed by glycine, serine, and glutamic acid, but not by histidine or arginine (Fig. 3A-C). The activity of CBS in the liver was significantly higher in the rats fed the $25 \mathrm{CM}$ diet than in those fed the $25 \mathrm{C}$ diet, and enzyme activity was significantly decreased by glycine and significantly increased by glutamic acid (Fig. 3D).

The metabolism of methionine is strictly regulated by several mechanisms, ${ }^{9}$ ) but regulation of methionine metabolism appeared to be disrupted in the rats fed a high methionine diet, such as 25CM diet, which caused severe $(>100 \mu \mathrm{mol} / 1)^{1)}$ hyperhomocysteinemia. Milder
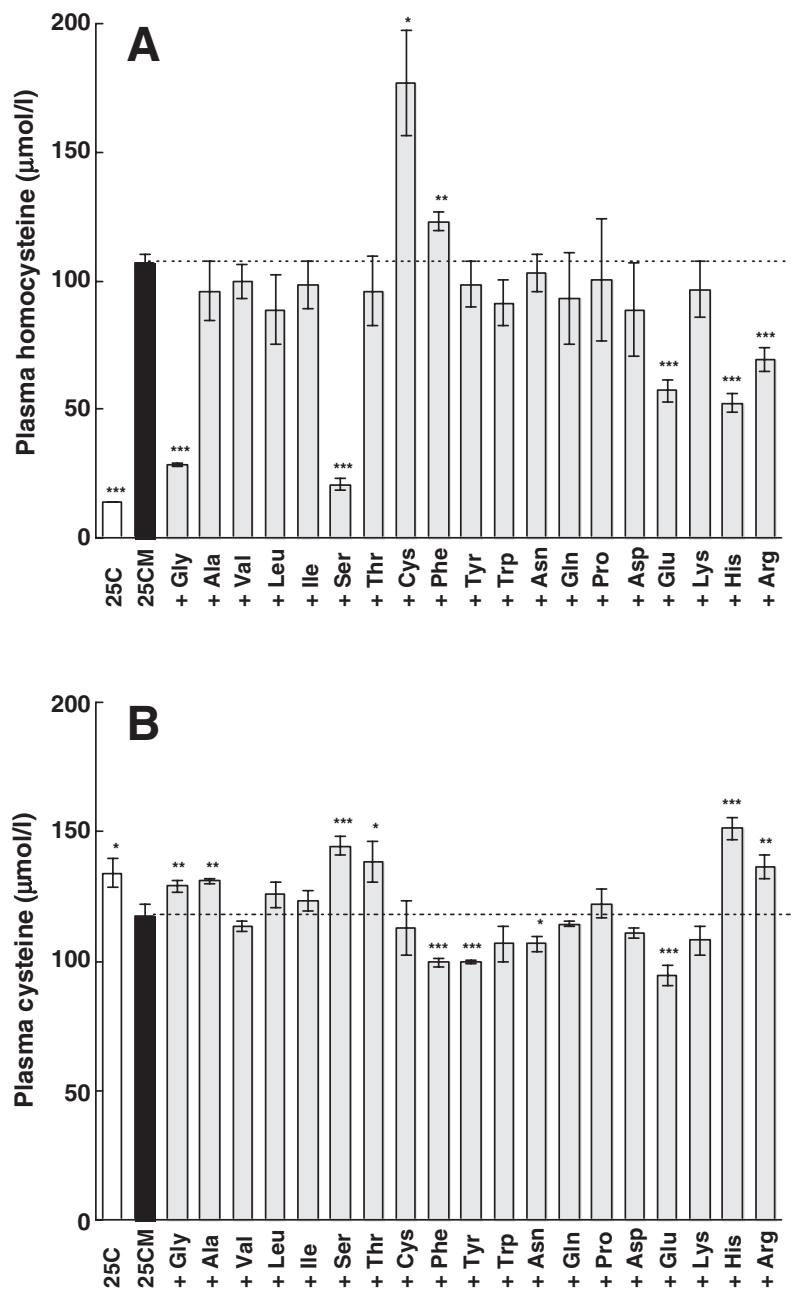

Fig. 2. Effects of Dietary Addition (2.5\%) of Various Kinds of Amino Acid on Plasma Homocysteine (A) and Cysteine (B) Concentrations in Rats Fed the 25\% Casein Diet Supplemented with $1 \%$ Methionine.

Each value is the mean \pm SEM for 5 (25C), 10 (25CM), or 4 (other groups) rats. $25 \mathrm{C}, 25 \%$ casein diet; $25 \mathrm{CM}, 25 \mathrm{C}+1 \% \mathrm{~L}-$ methionine diet. $*, * *, * * *$ Significantly different from the $25 \mathrm{CM}$ group at $p<0.05, p<0.01$, and $p<0.001$ respectively.

conditions might be appropriate in assessing the effects of amino acids on the plasma homocysteine concentration in terms of nutritional significance, but we used the $25 \mathrm{CM}$ diet in the present study, since glycine and serine had a strong hypohomocysteinemic effect even in the rats fed the $25 \mathrm{CM}$ diet. $^{4}$ )

The present study indicates that methionine-induced enhancement of the plasma homocysteine concentration can be significantly suppressed by the concurrent addition of glutamic acid, histidine, or arginine. Although homocysteine has two metabolic outcomes, i.e., remethylation and cystathionine formation, the latter is thought to be critical in the effective metabolism of homocysteine under conditions of methionine loading. ${ }^{10)}$ Previously, we found that glycine and serine elicited their effects mainly by stimulating cystathionine formation due to the supply of serine. ${ }^{4)}$ The result that methionine-induced increases in the hepatic concentra- 

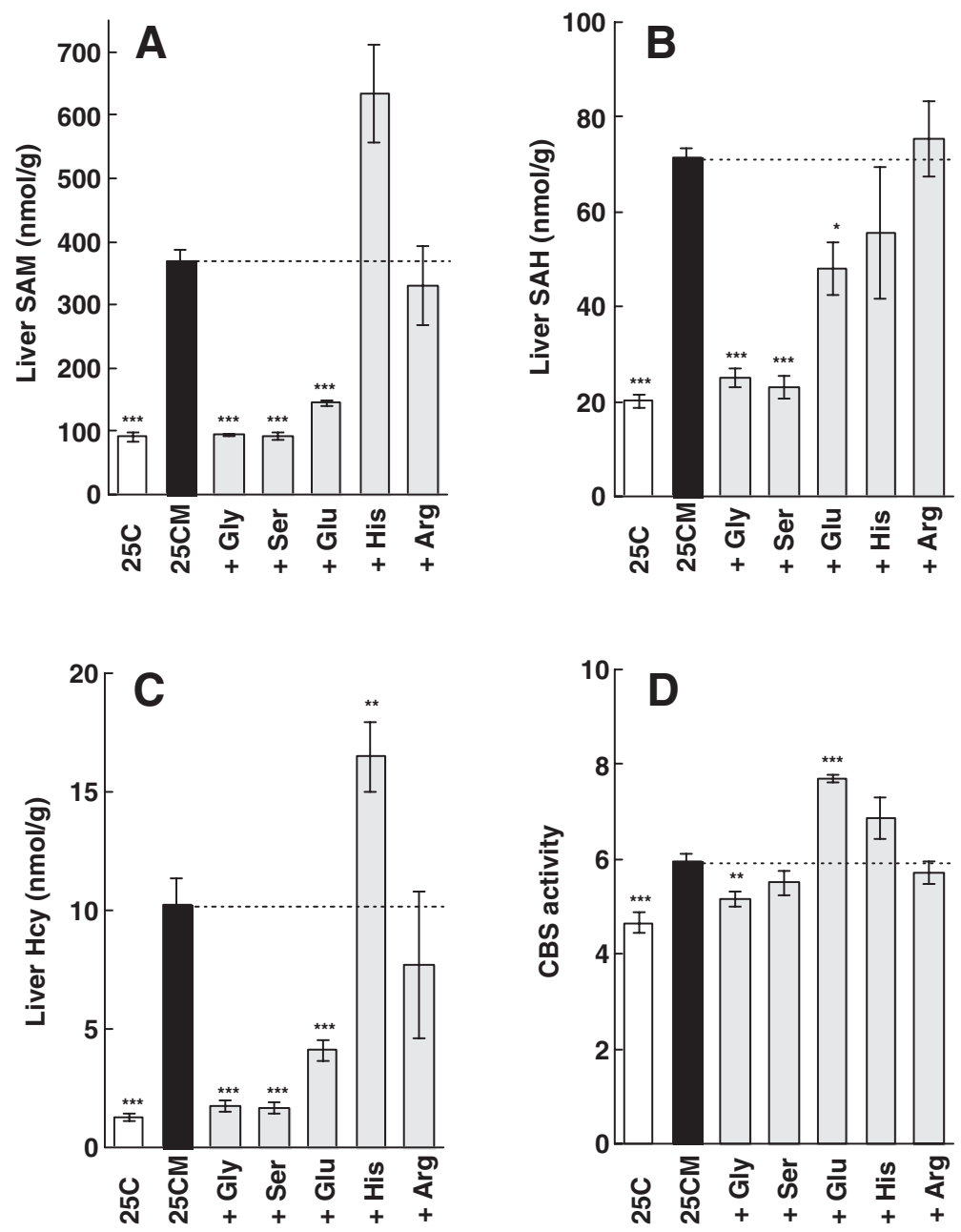

Fig. 3. Effects of Dietary Addition (2.5\%) of Certain Amino Acids on the Hepatic Concentrations of $S$-Adenosylmethionine (A), $S$-Adenosylhomocysteine (B), and Homocysteine (C), and the Activity of Cystathionine $\beta$-Synthase (D) in Rats Fed the $25 \%$ Casein Diet Supplemented with $1 \%$ Methionine.

Each value is the mean $\pm \mathrm{SEM}$ for 5 (25C), 10 (25CM), or 4 (other groups) rats. $25 \mathrm{C}, 25 \%$ casein diet; $25 \mathrm{CM}, 25 \mathrm{C}+1 \% \mathrm{~L}-\mathrm{methionine}$ diet; CBS, cystathionine $\beta$-synthase. Cystathionine $\beta$-synthase activity was expressed as $\mathrm{nmol} / \mathrm{min} / \mathrm{mg}$ of protein. ${ }^{*, * * * * *}$ Significantly different from the 25CM group at $p<0.05, p<0.01$, and $p<0.001$ respectively.

tions of methionine metabolites were suppressed by glutamic acid in addition to glycine and serine suggests that glutamic acid also stimulated the metabolism of homocysteine and methionine. A significant increase in hepatic CBS activity in rats fed the glutamic acid-added diet might be partly associated with the hypohomocysteinemic effect of glutamic acid, but the detailed mechanism underlying the effect of glutamic acid remains to be fully elucidated.

On the other hand, the effects of histidine and arginine on hepatic concentrations of methionine metabolites were different from the effects of glycine, serine, and glutamic acid, suggesting that the mechanisms by which histidine and arginine elicited their effects are different from those of glycine, serine, and glutamic acid. It has been found that methionine supplementation enhances the catabolism of histidine in rats, ${ }^{11,12)}$ whereas little information is available concerning the effect of histidine on methionine metabolism. Histidine is catabolized to provide the formimino group, which is accepted by tetrahydrofolate and converted to various forms of onecarbon unit, including 5- $\mathrm{CH}_{3}$-tetrahydrofolate. In fact, Kohashi et al. ${ }^{13)}$ have found that the hepatic concentration of 5- $\mathrm{CH}_{3}$-tetrahydrofolate was markedly higher in rats fed a histidine-excess diet than in rats fed a control diet. Since homocysteine can be remethylated to methionine using the methyl group of $5-\mathrm{CH}_{3}$-tetrahydrofolate or betaine, it is possible that dietary addition of histidine increased 5- $\mathrm{CH}_{3}$-tetrahydrofolate-dependent remethylation of homocysteine, and thereby decreased the plasma homocysteine concentration. However, histidine addition did not suppress methionine-induced elevation of the hepatic homocysteine concentration, raising a question about the plasma homocysteinelowering effects of histidine. Further study is needed to confirm the effect of histidine. It has been reported that dietary addition of arginine increases the production of nitric oxide in rats ${ }^{14)}$ and that nitric oxide reacts with 
homocysteine to form nitroso-homocysteine. ${ }^{15)}$ Therefore, one possible mechanism of the effect of arginine is that dietary addition of arginine increases nitric oxide production, thereby stimulating the formation of nitrosohomocysteine and leading to a decrease in the plasma homocysteine concentration. In support of this, Faldetta et al. ${ }^{16)}$ have found that acute intraperitoneal infusion of L-arginine results in a transient decrease in the plasma total homocysteine concentration in diabetic and healthy subjects.

In addition to effects on the metabolism of methionine and homocysteine, there is a possibility that the amino acids, which suppressed the methionine-induced enhancement of plasma homocysteine concentration, inhibited methionine transport in the intestine and other tissues and thereby elicited their effects, since methionine shares transporters with several other amino acids. ${ }^{17)}$ On the other hand, the plasma cysteine concentration was significantly increased by several amino acids, including glycine, serine, histidine, and arginine (Fig. 2B). Cysteine competes with homocysteine for transporters in several tissues ${ }^{18)}$ and for the homocysteine-binding site of plasma albumin. ${ }^{19)}$ Thus, the possibility that the increase in the plasma cysteine concentration is partly associated with the effects of glycine, serine, histidine, and arginine cannot be excluded, although the causal relationship is still unclear.

It has been reported that cystine and $N$-acetylcysteine have hypohomocysteinemic effects in humans when administered as a single meal or a single dose. ${ }^{3,19)}$ In contrast, dietary addition of cysteine did not decrease, but rather increased, the plasma homocysteine concentration in the present study. The reason for the discrepancy between the results of previous studies and the results of this one appears at least in part to be different experimental conditions, e.g., the addition level and administration method of methionine and cysteine. One possible mechanism of the hyperhomocysteinemic effect of cysteine at a relatively high level in rats fed a high methionine diet is the repression of CBS by cysteine. ${ }^{20)}$

\section{References}

1) Refsum, H., Ueland, P. M., Nygard, O., and Vollset, S. E., Homocysteine and cardiovascular disease. Annu. Rev. Med., 49, 31-62 (1998).

2) Selhub, J., Homocysteine metabolism. Annu. Rev. Nutr., 19, 217-246 (1999).

3) Verhoef, P., Steenge, G. R., Boelsma, E., van Viet, T., Olthof, M. R., and Katan, M. B., Dietary serine and cystine attenuate the homocysteine-raising effect of dietary methionine: a randomized crossover trial in humans. Am. J. Clin. Nutr., 80, 674-679 (2004).

4) Fukada, S., Shimada, Y., Morita, T., and Sugiyama, K., Suppression of methionine-induced hyperhomocysteinemia by glycine and serine in rats. Biosci. Biotechnol. Biochem., 70, 2403-2409 (2006).

5) Durand, P., Fortin, L. J., Luissier-Cacan, S., Davignon, J., and Blache, D., Hyperhomocysteinemia induced by folic acid deficiency and methionine load-application of a modified HPLC method. Clin. Chim. Acta, 252, 83-93 (1996).

6) Cook, R. J., Horne, D. W., and Wagner, C., Effect of dietary methyl group deficiency on one-carbon metabolism in rats. J. Nutr., 119, 612-617 (1988).

7) Mudd, S. H., Finkelstein, J. D., Irreverre, F., and Laster, L., Transsulfuration in mammals: microassay and tissue distributions of three enzymes of the pathway. J. Biol. Chem., 240, 4382-4392 (1965).

8) Einarsson, S., Josefsson, B., and Lagerkvist, S., Determination of amino acids with 9-fluorenylmethyl chloroformate and reversed-phase high-performance liquid chromatography. J. Chromatogr., 282, 609-618 (1983).

9) Stipanuk, M. H., Sulfur amino acid metabolism: pathways for production and removal of homocysteine and cysteine. Annu. Rev. Nutr., 24, 537-577 (2004).

10) Finkelstein, J. D., and Martin, J. J., Methionine metabolism in mammals: adaptation to methionine excess. J. Biol. Chem., 261, 1582-1587 (1986).

11) Krebs, H. A., Hemes, R., and Tyler, B., The regulation of folate and methionine metabolism. Biochem. J., 158, 341-353 (1976).

12) Fell, D., and Steele, R. D., Effect of methionine on in vivo histidine metabolism in rats. J. Nutr., 113, 860-866 (1983).

13) Kohashi, M., Takahashi, A., and Iwai, K., Effect of a histidine-excess diet on a tetrahydrofolylpolyglutamate pattern in rat liver. J. Nutr. Sci. Vitaminol., 36, 11-19 (1990).

14) Fu, W. J., Haynes, T. E., Kohli, R., Hu, J., Shi, W., Spencer, T. E., Carroll, R. J., Meininger, C. J., and Wu, G., Dietary L-arginine supplementation reduces fat mass in Zucker diabetic fatty rats. J. Nutr., 135, 714-721 (2005).

15) Stamler, J. S., Osborne, J. A., Jaraki, O., Rabbani, L. E., Mullins, M., Singel, D., and Loscalzo, J., Adverse vascular effects of homocysteine are modulated by endothelium-derived relaxing factor and related oxides of nitrogen. J. Clin. Invest., 91, 308-318 (1993).

16) Faldetta, M. C., Laurenti, O., Desideri, G., Bravi, M. C., De Luca, O., Marinucci, M. C., De Mattia, G., and Ferri, C., L-Arginine infusion decreases plasma total homocysteine concentrations through increased nitric oxide production and decreased oxidative status in type II diabetic patients. Diabetologia, 45, 1120-1127 (2002).

17) Mann, G. E., Yudilevich, D. L., and Sobrevia, L., Regulation of amino acid and glucose transporters in endothelial and smooth muscle cells. Physiol. Rev., 83, 183-252 (2003).

18) Budy, B., O'Neill, R., Dibello, P. M., Sengupta, S., and Jacobsen, D. W., Homocysteine transport by human aortic endothelial cells: identification and properties of import system. Arch. Biochem. Biophys., 446, 119-130 (2006).

19) Hultberg, B., Andersson, A., Masson, P., Larson, M., and Tunek, A., Plasma homocysteine and thiol compound fraction after oral administration of $N$-acetylcysteine. Scand. J. Clin. Lab. Invest., 54, 417-422 (1994).

20) Finkelstein, J. D., and Mudd, H., Trans-sulfuration in mammals: the methionine-sparing effect of cystine. $J$. Biol. Chem., 242, 873-880 (1967). 